\title{
Patents-4-Patients - The Cancer Moonshot gets EXTENDED!
}

\section{Joseph E Kovarik* \\ Sheridan Ross, Colorado, USA}

The cure for cancer is perhaps the proverbial long-felt but unsolved problem of mankind. While steady progress has been made over the decades, it has been relatively slow and frustrating. Recent immunotherapies seem to offer the best promise of a viable treatment - but many questions remain. The importance of research and development in this area is undeniable. But the costs associated with such efforts are enormous and will simply not be undertaken by the private sector without the existence of some significant commercial upside to counterbalance the considerable risks of failure. The only viable way to offer some protection for inventions in this area lies in obtaining meaningful patent protection. The process and time involved in obtaining patent protection, however, is significant, with average patent applications taking at least 3 or more years to issue - if they issue at all.

Investing in purported cancer cures without any assurances that patent protection will be available is a non-starter for seemingly otherwise promising research prospects. The Cancer Moonshot Program - aka - "Patents 4 Patients" was initiated under the Obama administration - providing an avenue for cancer researchers to obtain issued patents at unprecedented speed - and without the costs typically required for expedited patent examination by the US Patent and Trademark Office. Surprisingly, despite its planned expiration on December 31, 2018, the Cancer Moonshot Program has been extended until June 30, 2020. Every cancer researcher who is not already taking advantage of the Cancer Moonshot Program - should do so immediately.

President Kennedy, in a speech delivered at Rice University in 1962 said: "We choose to go to the moon. We choose to go to the moon in this decade and do the other things, not because they are easy, but because they are hard, because that goal will serve to organize and measure the best of our energies and skills, because that challenge is one that we are willing to accept, one we are unwilling to postpone, and one which we intend to win, and the others, too." Echoing this odd phrase, Joe Biden announced the Cancer Moonshot on June 29, 2016 with the words: "We should be unwilling to postpone finding the answer to how to end cancer as we know it. And I'm confident we can do it."

The "Cancer Immunotherapy Pilot Program" ("Patents4Patients") was initiated to support of the White House's \$1 billion initiative to achieve ten years' worth of cancer research in the next five. As outlined in the Federal Register of June 29, 2016, the program provides for earlier review of patent applications pertaining to cancer immunotherapy, completing examination with 12 months from filing. Normally, if one wanted such accelerated patent prosecution (under the Prioritized Examination (Track I) program), the additional filing fee would be $\$ 4,000$ (for an entity of 500 or more employees), and $\$ 2,000$ for a smaller entity. The fee for the Cancer Moonshot program is " $\$ 0$ " the only required fee is the normal filing/search and examination fees (\$800 for a small entity).

The program is open to regular utility patent applications with no more than 3 independent claims and 20 total claims. At least one claim must be directed to a method of treating cancer using immunotherapy. For example, at least one claim must specify it is encompassing a method of ameliorating, treating, or preventing a malignancy in a human subject wherein the steps of the method assist or boost the immune system in eradicating cancerous cells.

\section{Applicants must submit a petition and statements including:}

- A "Petition to Make Special" - requesting "special status" due to the fact the application includes a claim that meets the eligibility requirements;

- a statement that, should the USPTO find that the invention is directed to multiple inventions, the applicant agrees to make an election without traverse in a telephonic interview to elect an invention that is directed to a method of treating a cancer using immunotherapy;

- a statement that the application has not previously been granted special status under any other USPTO program; and

\section{- a request for early publication.}

The petition must be filed either at least one day before the first Office Action is issued or with a proper request for continued examination (RCE). If, however, the claimed cancer immunotherapy method is the subject of an active Investigational New Drug (IND) application that has entered phase II or III clinical trials, then a petition may be filed even after receiving a first office action from the USPTO (but prior to a final office action).

A few words of caution: any request for an extension of time will terminate the application's special status; and thus, there is only limited time ( 3 months) to provide experimental data/evidence to address any enablement or obviousness rejections. You can only submit 3 independent claims and a total of 20 claims and importantly, at least one claim must be directed to a method of treating cancer using immunotherapy. For example, eligible claims could include administration of cells, antibodies, proteins, or nucleic acids that invoke

${ }^{\star}$ Correspondence to: Joseph E Kovarik, Sheridan Ross, PC, 1560 Broadway \#1200, Denver, Colorado 80202, USA, E-mail: jkovarik@sheridanross.com

Received: December 21, 2018; Accepted: January 10, 2019; Published: January 14,2019 
an active immune response (or achieve a passive immune response to destroy cancerous cells); combination therapies of biological adjuvants and conventional therapies for treating cancer, vaccines that activate the immune system to prevent or destroy the growth of cancer cells. In vivo, ex vivo, and adoptive immunotherapies will also be considered. Finally, the Cancer Moonshot Program ends Dec. 31, 2018- so urgent action is suggested.

In contrast to the Patents 4 Patients program, conventional USPTO examination takes a long time - first office action pendency (i.e., how long it takes to mail a First Office Action, which is overwhelmingly a rejection of all pending claims) after a patent application is filed, takes an average of 16.2 months, and traditional total pendency, or how long it takes to decide whether to issue or abandon a patent (not including the additional one to four months for issuance), takes an average of 24.5 months. And as referenced earlier, fees associated with fast-tracking a patent can be cost-prohibitive. The "Patents 4 Patients" program eliminates both the time and cost barriers.

Thus far, the Cancer Moonshot has been a resounding success. Recent data from the USPTO indicates that $91 \%$ of submissions have been granted, e.g. of the 228 total submissions, 208 were granted and only 20 were dismissed. About $2 / 3$ rds of the cases were closed within a year, with another $1 / 3^{\text {rd }}$ pending. Over 80 patents have issued under the Program. Learn more about the program on the US Patent \& Trademark Office website here: https://www.uspto.gov/about-us/ national-cancer-moonshot. The take-home point:Hurry - this is a rare "sale" by the USPTO that is only available for cancer researchers. What traditionally has taken over 3-5 years under the US patent system, can now be achieved in less than 12 months.

Copyright: (C2019 Kovarik JE. This is an open-access article distributed under the terms of the Creative Commons Attribution License, which permits unrestricted use, distribution, and reproduction in any medium, provided the original author and source are credited. 